\title{
Polimorfismo del gen de la tiopurina S-metiltransferasa en donantes de sangre de un hospital universitario
}

\author{
Luis Álvarez $L^{1}$, Mauricio Venegas $S^{1 a}$, Milton Larrondo $L^{2}$, \\ Natalia Becerra B ${ }^{1 b}$, Ariel Castro $L^{3 c}$, Rodrigo Q uera $\mathrm{P}^{1,4}$. \\ Thiopurine S-methyltransferase gene \\ polymorphism in Chilean blood donors
}

Background: Thiopurine S-methyltransferase (TPMT) is a cytosolic enzyme that catalyzes the S-methylation of 6-mercaptopurine and azathioprine. Lowactivity phenotypes are correlated with polymorphism in the TPMT gene. Patients with low or undetectable TMPT activity could develop severe myelosuppression when they are treated with standard doses of thiopurine drugs. Since ethnic differences in the TPMT gen polymorphism have been demonstrated worldwide, its assessment in the Chilean population is worthwhile. Aim: To investigate the TMPT gene polymorphism in a Chilean blood donor individuals. Subjects and Methods: The frequency of four allelic variants of the TPMT gene, *2 (G238C), *3A (G460A and A719G), *3B (G460A) and *3C (A719G) were analyzed in 210 Chilean blood donors, using polymerase chain reaction (PCR), restriction fragment length polymorphism (RFLP) and allele-specific PCR-based assays. Results: TPMT variants associated to low enzymatic activity, were detected in 16 subjects (8\%), who had a heterozygous genotype (*3A in 12 ; ${ }^{3} \mathrm{C}$ in three and $* 2$ in one subject). No TPMT*3B allelic variant was found. The normal allele (wild-type) was found in 92\% of studied individuals. Conclusions: The allele TPMT*3A, is the most prevalent in this group of Chilean blood donors, as in Caucasian populations (Rev Méd Chile 2009; 137: 185-92).

(Key words: Blood donors; Genetic polymorphism; Methyltransferases)

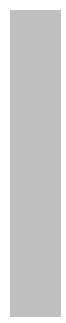

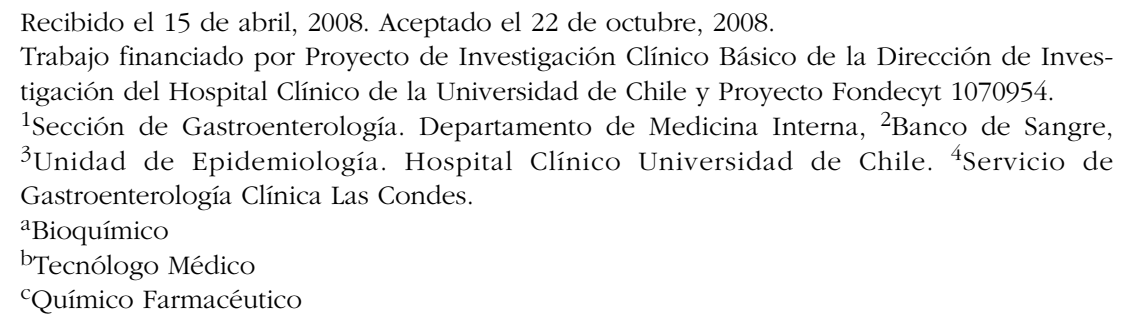

Correspondencia a: Dr. Rodrigo Quera P. Servicio de Gastroenterología, Clínica Las Condes. Lo Fontecilla 441. Santiago. E mail: rquera@clinicalascondes.cl 
$\mathrm{L}^{2}$ a tiopurina S-metiltransferasa (TMPT) representa uno de los ejemplos más notables de cómo la farmacogenética puede contribuir a individualizar un fármaco ${ }^{1}$. En este sentido, la respuesta al tratamiento con tiopurinas, ya sea azatioprina (AZA) o 6-mercaptopurina (6-MP), que corresponden a fármacos inmunomoduladores que se utilizan en el tratamiento de enfermedades autoinmunes ${ }^{2-5}$, leucemias ${ }^{6}$ y en trasplante de órganos ${ }^{7}$, se podría predecir genéticamente. La AZA es una prodroga que es convertida a 6-MP in vivo, siendo ésta también una prodroga que sufre una serie de reacciones enzimáticas para formar nucleótidos de tioguaninas (6-TGNs), metabolitos activos que pueden ser incorporados al ADN. Dos importantes reacciones inactivan a las drogas tiopurínicas in vivo, la oxidación catalizada por la xantinooxidasa y la S-metilación catalizada por TMPT (Figura 1). Debido a la ausencia de xantinooxidasa a nivel hematopoyético, la metilación por la TMPT tiene un rol preferencial en la metabolización de las drogas tiopurínicas ${ }^{8}$.
La existencia de polimorfismo en el gen de la TMPT genera variantes alélicas, cuyo fenotipo muestra una capacidad disminuida en la S-metilación de AZA/6-MP, determinando un aumento de los metabolitos activos (6-TGNs), lo que produce una mayor actividad farmacológica, pero también un mayor riesgo de efectos adversos. El gen que codifica para la TPMT se encuentra en el cromosoma 6p22.3, siendo sus alelos heredados de manera codominante y presenta variaciones genéticas que controlan la actividad enzimática9,10. Esta actividad enzimática variable influye en la biodisponibilidad de las drogas tiopurínicas, determinando su nivel de actividad clínica y el riesgo de efectos adversos dependientes de la dosis, dentro de las cuales destaca la mielosupresión. Se han publicado 21 variantes alélicas para el gen de la TPMT, siendo el alelo TPMT*1 el correspondiente al alelo wild type ${ }^{11-13}$, el cual es el más frecuente y cuyo fenotipo es de alta actividad enzimática. El resto corresponden a alelos asociados con menor actividad de TPMT, de los cuales los alelos
Figura 1. Metabolismo de las tiopurinas. La pro-droga azatioprina es convertida rápidamente, mediante una reacción no enzimática, a 6-mercaptopurina (6-MP), la cual es metabolizada por tres vías; xantinooxidasa (XO), tiopurina S-metiltransferasa (TPMT) e hipoxantina guanina fosforibosil transferasa (HPRT). La vía mediada por XO produce el metabolito inactivo ácido 6 - tioúrico (6-TU). La TPMT cataliza la producción de otro metabolito inactivo, la 6-metilmercaptopurina (6-MMP). La vía de la HPRT genera 6tioinosina monofosfato (6-TIMP). Una vez formada, la 6-TIMP puede ser transformada por tres vías; mediante las enzimas inosina5-monofosfato deshidrogenasa (IMPDH) y guanina monofosfato sintetasa (GMPS), genera nucleótidos de tioguanina (6-TGNs), por medio de la TPMT es metilada a ribonucleótidos de 6-metilmercaptopurina (6-MMPR) o puede ser fosforilada por la enzima Inosina trifosfato pirofosfatasa (ITPasa) para generar 6-tio-inosina trifosfato (6tio-ITP). Los metabolitos 6-MMPR, 6-tio-ITP y 6-TGNs son activos. Estos últimos pueden incorporarse al ADN.

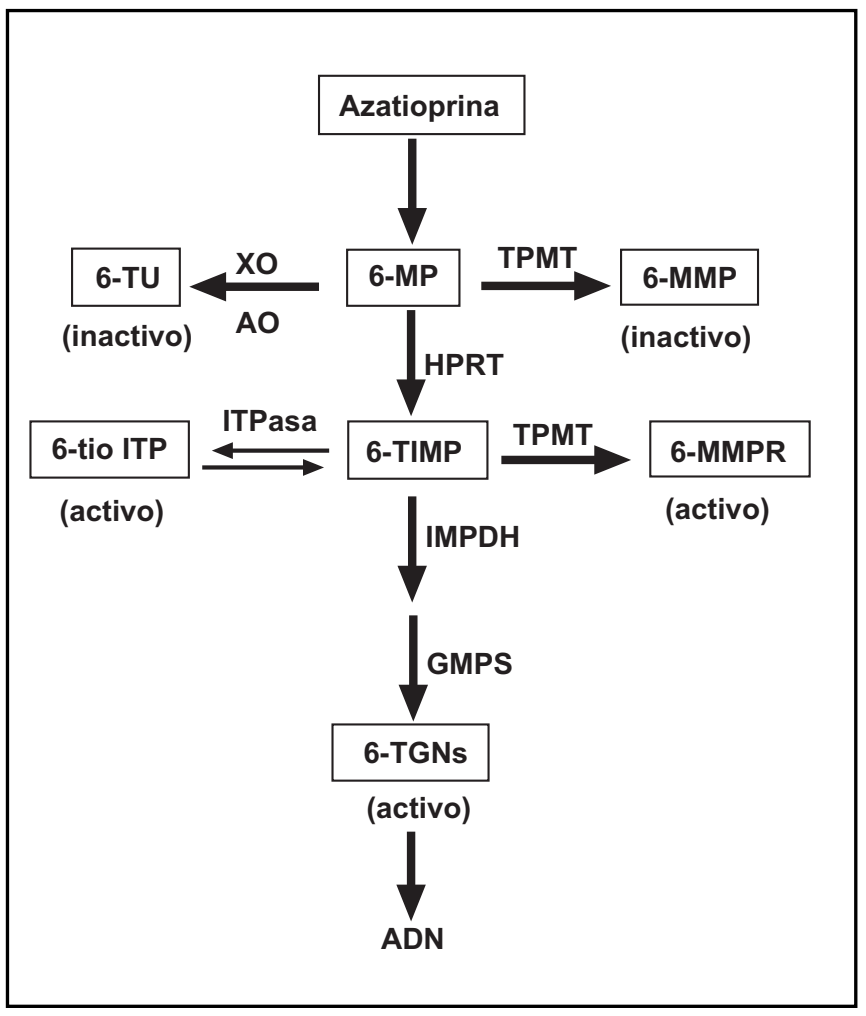


TPMT*2, *3A y *3C dan cuenta de más de 95\% de los casos publicados de la disminución de actividad de la TPMT y se generan como producto de polimorfismos de nucleótido único no sinónimos $(\mathrm{SNPs})^{14}$. La variante TPMT*2 corresponde a una transversión $\mathrm{G}^{238} \rightarrow \mathrm{C}$, que causa la sustitución Ala80Pro. La variante TPMT*3A contiene dos transiciones, $\mathrm{G}^{460} \rightarrow \mathrm{A}$ y $\mathrm{A}^{719} \rightarrow \mathrm{G}$, que resultan en las sustituciones Ala154Thr y Tyr240Cys, respectivamente. Para el alelo TPMT*3B se observa sólo la transición $\mathrm{G}^{460} \rightarrow \mathrm{A}$ (Ala154Thr) y en el caso del alelo TPMT*3C existe sólo la transición $A^{719} \rightarrow G$ (Tyr240Cys).

Estudios epidemiológicos han demostrado diferencias en las frecuencias alélicas de las distintas variantes del gen de la TPMT según el origen étnico de las poblaciones. Estudios realizados en Latinoamérica muestran una mayor frecuencia de alelo TPMT*3A en sujetos de Argentina ${ }^{15}$, Colombia $^{16}$ y Bolivia ${ }^{17}$, mientras que en la población de Brasil el alelo más frecuente corresponde al TPMT* $2^{18}$. Hasta la fecha no existen estudios sobre el polimorfismo del gen de la TPMT en población chilena. El objetivo de este trabajo es determinar la presencia y prevalencia de los alelos de la TPMT en una población de dadores de sangre.

\section{MATerial y MÉTODO}

Muestras. Se estudiaron, previo consentimiento informado, 210 muestras de sangre de donantes del Hospital Clínico de la Universidad de Chile. La edad promedio fue de 34 años, rango 19-68 años, 84 mujeres y 126 hombres. Todos los individuos incluidos en este estudio provienen de familias nacidas en Chile en sus dos últimas generaciones. Las muestras fueron recibidas en tubos con EDTA como anticoagulante y congeladas a $-20^{\circ} \mathrm{C}$ hasta su procesamiento.

Detección de polimorfismos. Se trabajó con ADN genómico obtenido a partir de las muestras de sangre. Para el aislamiento del ADN se utilizó el kit comercial Wizard ${ }^{\circledR}$ Genomic DNA purification (Promega, Madison WI, USA). Los genotipos de TPMT fueron determinados mediante metodologías de PCR descritas previamente ${ }^{19,20}$. Estos análisis utilizan pares de partidores que incluyen secuencias intrónicas y exónicas para asegurar la amplificación del gen de TPMT y no de un pseudogen existente en el cromosoma 18q21.1.

Detección de G238C. Se realizó PCR alelo específico. En una reacción se utilizaron los partidores P2W (5'-GTATGATTTTAT GCAGGTTTG-3') y P2C (5'-TAAATAGGAACCATCGGACAC-3') para amplificar un segmento de 256 pares de bases (pb) del exón $\mathrm{V}$ del gen normal de la TPMT y en otra reacción se utilizaron los partidores $\mathrm{P} 2 \mathrm{M}$ (5'GTATGATTTTATGCAGGTTTC-3') y P2C, los cuales amplifican un segmento de igual tamaño de la variante que lleva la transversión. Para un volumen de reacción de $30 \mu \mathrm{L}$, se utilizaron $10 \mu \mathrm{L}$ de ADN genómico aislado y las concentraciones finales de los reactivos fueron las siguientes: Tris$\mathrm{HCl} 10 \mathrm{mM}$ (pH 9), KCl $50 \mathrm{mM}$, Triton ${ }^{\circledR} \mathrm{X}-100$ $0,1 \%, \mathrm{MgCl}_{2} 1,5 \mathrm{mM}$, dNTP's $0,2 \mathrm{mM}$, partidores $0,5 \mu \mathrm{M}$ y 1 unidad de Taq ADN polimerasa (Promega, Madison WI, USA).

La reacción de amplificación se llevó a cabo en el Termociclador PTC-100 (MJ Research) programado con 40 ciclos a $94^{\circ} \mathrm{C}$ por $40 \mathrm{~s}, 55^{\circ} \mathrm{C}$ por $40 \mathrm{~s}$ y $72^{\circ} \mathrm{C}$ por $1 \mathrm{~min}$, con una extensión final de 10 $\min$ a $72^{\circ} \mathrm{C}$.

Detección de G460A. Se realizó PCR y RFLP. En el PCR se amplificó un segmento de 365 pb del exón VII del gen de la TPMT. Para ello, se utilizaron los partidores P460F (5'-ATAACAGAGTGGGGAGGCTGC-3') y P460R (5'-CTAGAACCCAGAAAAAGTATAG-3'). Para el RFLP, $20 \mu \mathrm{L}$ del amplicón se incubaron por $1 \mathrm{~h}$ a $60^{\circ} \mathrm{C}$ en presencia de $5 \mathrm{U}$ de la enzima de restricción Mwo I (New England Biolabs, Inc. Beverly. MA, USA). Si no existe la mutación, la enzima corta en un sitio el $\mathrm{ADN}$, generando 2 fragmentos, uno de 267 y otro de $98 \mathrm{pb}$. La presencia de la sustitución G460A elimina el sitio de corte, por lo tanto el amplificado queda del tamaño original.

Detección de A719G. Se realizó PCR-RFLP. En el PCR se amplificó un segmento de 236 pb del exón $\mathrm{X}$ del gen de TPMT. Para ello se utilizó los partidores P719F (5'- AATCCCTGATGTCATTCTTCATAGTATTT-3') y P719R (5'-CAGGCTTTAGCATAATTTTCAATTCCTC-3'). Para el RFLP, $20 \mu \mathrm{L}$ del amplicón se incubaron por $2 \mathrm{~h}$ a $37^{\circ} \mathrm{C}$ en presencia de $5 \mathrm{U}$ de la enzima de restricción Acc I (New England Biolabs, Inc. Beverly, MA, USA). Si 
no existe la mutación, la enzima no corta el ADN amplificado, quedando del tamaño original. La presencia de la sustitución A719G genera un sitio de restricción, lo que da origen a 2 fragmentos, uno de 150 y otro de 86 pb.

Para todas las reacciones antes señaladas, las detecciones de los productos amplificados y de los fragmentos de restricción fueron detectados en gel de agarosa teñido con bromuro de etidio, visualizados con luz ultravioleta y fotografiados.

Estadística. Para el cálculo de tamaño muestral, se utilizó un universo de 200 donantes de sangre que mensualmente acude al banco de sangre. Con este universo, para una prevalencia de variación del factor $\alpha$ estudiar de 5\%, y un error de 5\%, el tamaño muestral requerido era de 139 sujetos.

Se utilizó la prueba de $\chi^{2}$ para establecer si la población de dadores de sangre se encontraba en equilibrio genético de Hardy-Weinberg para el locus TPMT. Mediante el uso del sistema ABO y Rh como marcadores genéticos, se estimó el porcentaje de mezcla caucásica de la muestra poblacional. Para ello se utilizó los datos de frecuencias publicadas para población española ${ }^{21}$ : ABO $* \mathrm{O}=0,65 ; \mathrm{RHD}^{*} \mathrm{~d}=0,41$ y aborígenes chile$\operatorname{nos}^{22,23}: \mathrm{ABO}^{*} \mathrm{O}=0,98 ; \mathrm{RHD}^{*} \mathrm{~d}=0,0$.

\section{Resultados}

De los 210 sujetos estudiados, 16 presentaban alguna de las variantes alélicas mencionadas (7,6\%), todos con carácter heterocigoto, siendo de estos 9 hombres y 7 mujeres. En las Tablas 1 y 2 se muestran las frecuencias genotípicas y alélicas, respectivamente. Como se puede observar, el genotipo wild-type es el más frecuente, seguido por la variante TPMT*3A. Ningún individuo presentó la variante TMPT*3B.

Tabla 1. Frecuencias genotípicas de las variantes del gen de la T PM T en una población de 210 donantes de sangre de un hospital universitario

\begin{tabular}{|lcc|}
\hline Genotipo & $\begin{array}{c}\text { Número de } \\
\text { individuos }\end{array}$ & $\begin{array}{c}\text { Frecuencia } \\
\% \pm \mathbf{I C}(\mathbf{9 5} \%)\end{array}$ \\
\hline TPMT $^{* 1}$ / TPMT*1 & 194 & $92,38 \pm 3,59$ \\
TPMT $^{*}$ / TPMT*2 & 1 & $0,48 \pm 0,93$ \\
TPMT*1 / TPMT*3A & 12 & $5,71 \pm 3,14$ \\
TPMT*1 / TPMT*3C & 3 & $1,43 \pm 1,6$ \\
Total & 210 & 100 \\
\hline
\end{tabular}

IC $=$ intervalo de confianza

Tabla 2. Frecuencias alélicas de las variantes del gen de la T PM T en una población de $\mathbf{2 1 0}$ donantes de sangre de un hospital universitario

\begin{tabular}{|lcc|}
\hline Alelo & $\begin{array}{c}\text { Número } \\
\text { de alelos }\end{array}$ & $\begin{array}{c}\text { Frecuencia } \\
\% \pm \text { IC (95\%) }\end{array}$ \\
\hline TPMT*1 & 404 & $96,19 \pm 1,83$ \\
TPMT*2 & 1 & $0,24 \pm 0,47$ \\
TPMT*3A & 12 & $2,86 \pm 1,59$ \\
TPMT*3C & 3 & $0,71 \pm 0,81$ \\
Total & 420 & 100 \\
\hline
\end{tabular}

IC = intervalo de confianza 
La distribución genotípica obtenida no se aparta de la esperada en equilibrio de HardyWeinberg, considerando dos alelos, el wild-type (TPMT*1) y el conjunto de los otros (no-TPMT*1). Los valores esperados para TPMT*1/TPMT*1, TPMT*1/noTPMT*1 y noTPMT*1/noTPMT*1 eran de 194,$3 ; 15,4$ y 0,3 .

El porcentaje de mezcla caucásica de la población estudiada, calculada en base a los sistemas ABO y Rh fue de $68,85 \%$. Su complemento $31,15 \%$, corresponde a componente indígena.

En la Tabla 3 se compara la frecuencia alélica de TPMT de nuestra serie con aquellas publicadas para otras poblaciones ${ }^{24}$, obteniendo porcentajes similares a las poblaciones caucásicas.

\section{Discusión}

El interés en el estudio de las variantes alélicas de la TPMT está dado por el uso cada vez más frecuente de AZA/6-MP en el manejo de pacientes con enfermedad inflamatoria intestinal ${ }^{5}$, así como su uso en otras patologías como la leucemia linfoblástica aguda infantil, hepatitis autoinmune, artritis reumatoide, miastenia gravis y trasplante de órganos ${ }^{2-4,6,7}$. En este contexto, los efectos adversos descritos posterior a la administración de estas drogas han sido asociados a la presencia de polimorfismo del gen de la TPMT y que obliga a la suspensión del tratamiento en 15-30\% de los pacientes ${ }^{25}$.

Tabla 3. Frecuencia de las variantes del gen de la T PMT en distintas poblaciones. Adaptado de Referencia 24

\begin{tabular}{|c|c|c|c|c|c|}
\hline Población & $\mathbf{N}$ & $\begin{array}{l}\text { TPMT}^{*} 2 \\
(\% \pm \text { EE })\end{array}$ & $\begin{array}{c}\left.\text { TPMT*3A }^{*} \text { ( } \% \pm \mathrm{EE}\right)\end{array}$ & $\begin{array}{l}\text { TPMT*3B }^{*}(\% \pm \mathrm{EE})\end{array}$ & $\begin{array}{c}\text { TPMT*3C }^{*}(\% \pm \mathrm{EE})\end{array}$ \\
\hline Caucásica americana & 282 & $0,2 \pm 0,27$ & $3,2 \pm 1,05$ & ND & $0,2 \pm 0.27$ \\
\hline Caucásica británica & 398 & $0,5 \pm 0,35$ & $0,45 \pm 0,34$ & ND & $0,3 \pm 0,27$ \\
\hline Caucásica francesa & 382 & $0,5 \pm 0,36$ & $5,7 \pm 1,9$ & ND & $0,8 \pm 0,46$ \\
\hline Caucásica francesa & 938 & $0,7 \pm 0,27$ & $3 \pm 0,56$ & 0 & $0,4 \pm 0,21$ \\
\hline Caucásica alemán & 2,428 & $0,2 \pm 0,09$ & $4,4 \pm 0,42$ & 0 & $0,4 \pm 0,13$ \\
\hline Caucásica polaca & 716 & $0,4 \pm 0,24$ & $2,7 \pm 0,16$ & 0 & $0,1 \pm 0,12$ \\
\hline Caucásica sueca & 1,600 & $0,06 \pm 0,06$ & $3,75 \pm 0,47$ & $0,13 \pm 0,09$ & $0,44 \pm 0,17$ \\
\hline Caucásica noruega & 132 & 0 & $3,4 \pm 1,58$ & 0 & $0,3 \pm 0,48$ \\
\hline Noruega saami & 388 & 0 & 0 & 0 & $3,3 \pm 0,91$ \\
\hline Caucásica bulgaria & 626 & $0,16 \pm 0,16$ & $2,24 \pm 0,59$ & 0 & $0,16 \pm 0,16$ \\
\hline Afro americana & 248 & $0,4 \pm 0,4$ & 0 & ND & $2,4 \pm 0,97$ \\
\hline Egipcia & 400 & 0 & $0,003 \pm 0,03$ & 0 & $0,013 \pm 0,06$ \\
\hline Ghana & 434 & 0 & 0 & ND & $7,6 \pm 1,27$ \\
\hline Keniana & 202 & 0 & 0 & ND & $5,4 \pm 1,59$ \\
\hline China & 384 & 0 & 0 & ND & $2,3 \pm 0,76$ \\
\hline China & 206 & 0 & 0 & 0 & $1 \pm 0,69$ \\
\hline China (niños) & 426 & 0 & 0 & 0 & $0,2 \pm 0,22$ \\
\hline Japonesa & 1,044 & 0 & 0 & 0 & $1,6 \pm 0,39$ \\
\hline Tailandés & 400 & 0 & 0 & 0 & $4,75 \pm 1,06$ \\
\hline Suroeste asiática & 198 & 0 & $1 \pm 0,71$ & ND & 0 \\
\hline Sureste asiástica & 1,098 & 0 & 0 & ND & $1 \pm 0,3$ \\
\hline Brasileña & 408 & $2,2 \pm 0,73$ & $1,5 \pm 0,6$ & $0,2 \pm 0,22$ & $1 \pm 0,49$ \\
\hline Española & 276 & ND & $3,26 \pm 1,07$ & $1,45 \pm 0,72$ & $1,45 \pm 0,72$ \\
\hline Argentina & 294 & $0,7 \pm 0,49$ & $2,1 \pm 0,84$ & 0 & 0 \\
\hline Colombiana & 280 & $0,4 \pm 0,38$ & $3,6 \pm 1,11$ & 0 & 0 \\
\hline Chilena HCUCh & 420 & $0,24 \pm 0,24$ & $2,86 \pm 0,81$ & 0 & $0,71 \pm 0,41$ \\
\hline
\end{tabular}

$\mathrm{N}=$ número de cromosomas. $\mathrm{EE}=$ error estándar 
Nuestro estudio es el primer trabajo descrito en extenso que evalúa la presencia de variantes alélicas de esta enzima en una población chilena. Recientemente, en un resumen presentado en un congreso, Jorquera y cols mostraron que $8 \%$ de los individuos donantes sanos presentaban variantes alélicas de la TPMT ${ }^{26}$. Nosotros encontramos la misma proporción; 16 de los $210(7,6 \%)$ sujetos incluidos presentaban alguna de las variantes alélicas, todas al estado heterocigoto. Porcentajes similares han sido descritos en Argentina ${ }^{15}$, Bolivia $^{17}$ y Colombia ${ }^{16}$

Existe una amplia variación individual en la capacidad de S-metilación de TPMT, lo que depende del tipo de alelo que tiene un individuo, ya sea al estado homo o heterocigoto ${ }^{19}$. Al respecto, mediante experimentos de expresión heteróloga, se ha descrito que la variante homocigota TPMT*2 posee una disminución de 100 veces en a capacidad de S-metilación ${ }^{27}$. En el caso de la variante TPMT*3A, si ésta es heredada en forma homocigota, existe una pérdida total de la actividad enzimática ${ }^{28}$. Por otro lado, los alelos del gen de la TPMT son heredados con un patrón autosómico codominante, por lo que la población heterocigota posee un nivel intermedio de actividad enzimática ${ }^{19}$.

Aunque 21 variantes alélicas del gen de la TPMT han sido identificadas, sólo tres de ellas han sido asociadas a cambios en la actividad de la TPMT (TPMT*2, *3A y *3C). La TPMT*3A es la variante genética más frecuente en la población caucásica, con una frecuencia que va desde 3,2\% a $5,7 \%$ dependiendo de la población incluida19,31-36. En la población asiática, la variante TPMT*3C es la más frecuente ${ }^{24}$. En Latinoamérica, en la población de Argentina ${ }^{15}$, Colombia ${ }^{16}$ y Bolivia ${ }^{17}$ predomina el alelo TPMT*3A, mientras que en Brasil $^{18}$, el alelo TPMT*3C es más común ${ }^{15}$. El polimorfismo TPMT*2 es una variante con una baja frecuencia alélica en la población caucásica, entre $0,17 \%$ a $0,5 \%{ }^{20}$. En nuestro estudio, la frecuencia alélicas de TMPT*3A, TPMT*3C y TPMT*2 fueron $2,86 \%, 0,71 \%$ y $0,24 \%$, respectivamente. Las diferencias en la frecuencia de las distintas variantes alélicas encontradas en los trabajos realizados en Sudamérica confirman que la composición étnica debe ser considerada al momento de evaluar la presencia de polimorfismos en una población.
Diversas publicaciones han demostrado un alto grado de correlación entre el genotipo y el fenotipo de la TPMT en población caucásica ${ }^{19,37}$. Jorquera y cols. encontraron que los individuos wild type tuvieron una actividad enzimática significativamente mayor que los heterocigotos, confirmando la correlación que existe entre genotipo-fenotipo ${ }^{26}$. Si bien nosotros no medimos la actividad enzimática de TPMT, todos los sujetos que presentaban alguna de las variantes alélicas eran de carácter heterocigoto, lo que podría predecir que tendrían una actividad intermedia.

La relación que existe entre la actividad de la TMPT y la respuesta al tratamiento o los efectos secundarios al uso de fármacos tiopurínicos, no es concluyente. En un estudio que incluyó 142 pacientes con enfermedad inflamatoria intestinal (97 con enfermedad de Crohn y 45 con colitis ulcerosa), la remisión se correlacionó con los niveles de 6-TGN (usando los niveles de 6-TGN como un marcador indirecto de actividad de la TPMT $^{38}$. En otro trabajo, realizado con 92 pacientes pediátricos, se demostró que la presencia de heterocigotos para alguna de las tres variantes TPMT*3, se asoció a concentraciones elevadas de 6-TGN y que todos respondieron al tratamiento 39 . Sin embargo, sólo uno de cada 13 pacientes que desarrollaron mielosupresión, presentaba esta variante alélica. Gisbert y cols. tampoco pudieron confirmar que la dosis de AZA/6-MP basado en la actividad de la TPMT pueda prevenir el riesgo de toxicidad medular ${ }^{40}$. Pese a la controversia que aún existe sobre la relevancia clínica del análisis de la actividad de la TPMT o el genotipo de esta enzima, parece razonable sugerir que si se conoce que un paciente tiene una actividad de la TPMT disminuida previo a la iniciación de la terapia, una dosis baja de AZT (1-1.5 mg/kg/día) puede ser útil hasta que la tolerancia y efectividad pueda ser demostrada.

Una de la razones para explicar porqué los resultados sobre la TPMT son tan contradictorios, se debe probablemente a que los estudios en general, han evaluado únicamente los polimorfismos en una de las enzimas de las vías de la metabolización de las tiopurinas. El metabolismo de las tiopurinas está dado por un número variado de vías y varios estudios han tratado últimamente de analizar otros polimorfismos incluidos en estas vías. Una de estas vías es la 
enzima inosina trifosfato pirofosfatasa (ITPasa) (Figura 1). La deficiencia de la ITPasa se asocia con el polimorfismo de un solo nucleótido que da lugar a un cambio en el aminoácido prolina por una treonina en el codón $32^{41}$. La presencia de esta variante alélica se ha asociado a la presencia de efectos adversos como mielosupresión, erupción cutánea y pancreatitis. Sin embargo, recientemente un metanálisis que incluyó seis estudios no pudo demostrar una correlación entre la presencia de este polimorfismo y el riesgo de toxicidad por tiopurinas, sugiriendo que la determinación de la ITPasa no posee ninguna relevancia clínica en pacientes tratados con AZA/6-MP ${ }^{42}$.

En conclusión, este trabajo confirma que las variantes alélicas de la TPMT encontradas en la población chilena estudiada son similares a las descritas en la población caucásica, aunque la muestra posee $31 \%$ de componente indígena.

\section{REFERENCIAS}

1. Krynetski EY, Tai HL, Yates CR, Fessing MY, LoenneCHEN T, SCHUETZ JD, ET AL. Genetic polymorphism of thiopurine S-methyltransferase: clinical importance and molecular mechanisms. Pharmacogenetics 1996; 6: 279-90.

2. Milanese C, La Mantia L, Salmaggi A, Eoli M. A double blind study of azathioprine efficacy in multiple sclerosis: final report. J Neurol 1993; 240: 295-8.

3. Kerstens PJ, Stolk JN, Boerbooms AM, Lambooy LH, De Graaf R, De Abreu RA. Purine enzymes in rheumatoid arthritis: possible association with response to azathioprine. A pilot study. Ann Rheum Dis 1994; 53: 608-11.

4. Heneghan ma, Allan ML, Bornstein JD, Muir A, TENDLER DA. Utility of thiopurine methyltransferase genotyping and phenotyping, and measurement of azathioprine metabolites in the management of patients with autoimmune hepatitis. J Hepatol 2006; 45: 584-91.

5. Derijks LJJ, Gilissen LPL, Hooymans PM, Hommes DW. Review article: thiopurines in inflammatory bowel disease. Aliment Pharmacol Ther 2006; 24: 715-29.

6. Lennard L, Lilleyman JS, Van Loon J, Weinshilboum RM. Genetic variation in response to 6-mercaptopurine for childhood acute lymphoblastic leukaemia. Lancet 1990; 336: 225-9.

7. Hollander aA, Van SaAse Jl, Kootte AM, Van Dorp WT, Van Bockel HJ, VAN Es LA, et al. Beneficial effects of conversion from cyclosporine to azathio-
Si nuestro estudio fuera extrapolable a gran parte de la población chilena, creemos que 7,6\% de genotipos que se asocian a baja actividad de TPMT, es un porcentaje alto y que debe ser considerado a la hora de indicar terapia con AZA/6-MP. Sin embargo, si bien la evaluación del polimorfismo de TPMT o la medición de la actividad enzimática pudiese alcanzar un papel en la práctica clínica y ser útil en la evaluación de pacientes que requieren tratamiento con AZA/6-MP para evitar el riesgo de toxicidad hematopoyética, esta estrategia no garantiza que estos eventos adversos no puedan ocurrir, por lo que una monitorización regular de los leucocitos y pruebas hepáticas es aún necesaria.

\section{Agradecimiento}

Al Dr. Carlos Valenzuela Yuraidini, Profesor Titular, Programa de Genética Humana, Instituto de Ciencias Biomédicas, Facultad de Medicina. Universidad de Chile; por su valiosa asesoría en conceptos de genética de poblaciones.

prine after kidney transplantation. Lancet 1995; 345 : 610-4.

8. Lennard L, Van Loon JA, Lilleyman JS, Weinshilboum RM. Thiopurine pharmacogenetics in leukemia: correlation of erythrocyte thiopurine methyltransferase activity and 6-thioguanine nucleotide concentrations. Clin Pharmacol Ther 1987; 41: 18-25.

9. Szumlanski C, Otterness D, Her C, Lee D, Brandriff B, KelSell D, ET AL. Thiopurine methyltransferase pharmacogenetics: human gene cloning and characterization of a common polymorphism. DNA Cell Biol 1996; 15: 17-30.

10. Seki T, Tanaka T, Nakamura Y. Genomic structure and multiple single-nucleotide polymorphisms (SNPs) of the thiopurine S-methyltransferase (TPMT) gene. J Hum Genet 2000; 45: 299-302.

11. Salavaggione OE, Wang L, Wiepert M, Yee VC, Weinshilboum RM. Thiopurine S-methyltransferase pharmacogenetics: variant allele functional and comparative genomics. Pharmacogenet Genomics 2005; 11: 801-15.

12. Schaeffler E, Fischer C, Brockmeier D, Wernet D, Moerike K, Eichelbaum M, et al. Comprehensive analysis of thiopurine S-methyltransferase phenotype-genotype correlation in a large population of German-Caucasians and identification of novel TPMT variants. Pharmacogenetics 2004; 7: 407-17.

13. Schaeffeler E, Eichelbaum M. Reinisch W, Zanger Um, SCWAB M. Three novel thiopurine S-methyltransferase allelic variants (TPMT*20, *21, *22) - association with decreased enzyme function. Hum Mutat 2006; 27: 976. 
14. Wang L, Weinshilboum R. Thiopurine S-methyltransferase pharmacogenetics: insights, challenges and future directions. Oncogene 2006; 25: 1629-38.

15. Larovere le, Dodelson De Kremer R, Lambooy lHJ, De ABREU RA. Genetic polymorphism of thiopurine Smethyltransferase in Argentina. Ann Clin Biochem 2003; 40: 388-93.

16. Isaza C, Henao J, López AM, Cacabelos R. Allelic variants of the thiopurine methyltransferase (TMPT) gene in the Colombian population. Methods Fin Exp Clin Pharmacol 2003; 25: 423-9.

17. Lu HF, Shin MC, Hsueh SC, Chen CM, Chang JY, Chang JG. Molecular analysis of the thiopurine S-methyltransferase alleles in Bolivians and Tibetans. J Clin Pharm Ther 2005; 30: 491-6.

18. Boson WL, Romano-Silva Ma, Correa H, Falcao RP, Teixeira-Vidigal Pv, De Marco L. Thiopurine methyltransferase polymorphisms in a Brazilian population. Pharmacogenomics J 2003; 3: 178-82.

19. Yates CR, Krynetski E, Loennechen T, Fessing M, Tal HL, PUi CH, ET AL. Molecular diagnosis of thiopurine S-Methyltransferase deficiency: Genetic basis for azathioprine and mercaptopurine intolerance. Ann Inter Med 1997; 126: 608-14.

20. Loennechen T, Utsi E, Hartz I, Lysaa R, Kildalsen H, Aarbakke J. Detection of one single mutation predicts thiopurine S-methyltransferase activity in a population of Saami in northern Norway. Clin Pharmacol Ther 2001; 70: 183-8.

21. Campillo FL. Estudio de los Grupos Sanguíneos en la Población Española. Comunicación de la Real Academia de Medicina. Tomo XCII de los Anales. Madrid: Cuaderno Tercero 1976

22. Matson A, Sutton E, Etcheverry R, Swanson J, Robinson A. Distribution of hereditary blood groups among Indians in South America. Am J Phys Anthropol 1967; 27: 157-94.

23. Llop E, Rothhammer F. A note on the presence of blood groups A y B in pre-Columbian South America. Am J Phys Anthropol 1988; 75: 107-11.

24. Sahasranaman S, Howard D, Roy S. Clinical pharmacology and pharmacogenetics of thiopurines. Eur J Clin Pharmacol 2008; 64: 753-67.

25. Fraser AG, Orchard TR, Jewell DP. The efficacy of azathioprine for the treatment of inflammatory bowel disease. A 30 year review. Gut 2000; 50: 485-9.

26. Jorquera A, Selman C, Vollrath V, Solari S, Álvarez M. Fenotipo y genotipo de la tiopurina metiltransferasa (TPMT) en población chilena. Gastr Latinoam 2006; 17: 447 (Abstract).

27. Krinetski Ey, Schuetz JD, Gapin AJ, Pui CH, Relling MV, Evans WE. A single mutation leading to loss of catalytic activity in human thiopurine s-methyltranferase. Proc Natl Acd Sci USA 1995; 92: 949-53.

28. Tai HL, Krinetski Ey, Yates CR, Loennechen T, Fessing M, KRYNETSKAIA NF ET AL. Thiopurine S-methyltranferase deficiency: two nucleotidee transitions define the most prevalent mutant allele associated with loss of catalytic activity in caucasians. Am J Hum Genet 1996; 58: 694-702.

29. Evans WE, Hon Yy, Bomgaars L, Coutre S, HoldsworTH M, JANCO R, ET AL. Preponderance of thiopurine Smethyltransferase deficiency and heterozygosity among patients intolerant to mercaptopurine or azathioprine. J Clin Oncol 2001; 19: 2293-301.

30. McLeod HL, Silva C. The thiopurine S-methyltransferase gene locus-implications for clinical pharmacogenomics. Pharmacogenomics 2002; 3: 89-98.

31. Krynetski Ey, Evans WE. Genetic polymorphism of thiopurine S-methyltransferase: molecular mechanism and clinical importance. Pharmacology 2000; 61: 136-46.

32. Schaeffler E, Lang T, Zanger Um, Eichelbaum M, SCHWAB M. High-throughput genotyping of thiopurine S-methyltransferase by denaturing HPLC. Clin Chem 2001; 47: 548-55.

33. Weinshilboum R. Thiopurine pharmacogenetics: clinical and molecular studies of thiopurine methyltransferase. Drug Metab Dispos 2001; 29: 601-5.

34. LENNARD L. TMPT in the treatment of Crohn's disease with azathioprine. Gut 2002; 51: 143-6.

35. Reuther lo, Sonne J, Larsen N, Dahlerup JF, Thomsen OO, Schmiegelow K. Thiopurine methyltransferase genotype distribution in patients with Crohn's disease. Aliment Pharmacol Ther 2003; 17: 65-8.

36. KurZawski M, Gawronska-Szkiarz B, DrozdZik M. Frequency distribution of thiopurine S-methyltransferase alleles in a Polish population. Ther Drug Monit 2004; 26: 541.5.

37. Rossi AM, Bianchi M, Guarnieri C, Barale R, Pacifici GM. Genotype-phenotype correlation for thiopurine S-methyltransferase in healthy Italian subjects. Eur J Clin Pharmacol 2001; 57: 51-4.

38. Cuffari C, Dassopoulos T, Turnbough L, Thompson Re, BAYLESS TM. Thiopurine methyltransferase activity influences clinical response to azathioprine in inflammatory bowel disease. Clin Gastroenterol Hepatol 2004; 2: 410-7.

39. Dubinsky MC, Lamothe S, Yang HY, Targan SR, Sinnett D, Theoret Y, et al. Pharmacogenomics and metabolite measurement for 6-mercaptopurine therapy in flmmatory bowel disease. Gastroenterology 2000; 118: 705-13.

40. Gisbert JP, Luna M, Mate J, González-Gujjarro L, Cara C, PAJARES JM. Choice of azathioprine or 6-mercaptopurine dose based on thiopurine methyltransferase (TPMT) activity to avoid myelosuppression. A prospective study. Hepatogastroenterology 2006; 53: 399-404.

41. Bierau J, Lindhout M, Bakker JA. Pharmacogenetic significance of inosine triphosphatase. Pharmacogenomics 2007; 8: 1221-8.

42. Van Dieren JM, Hansen BE, Kuipers EJ, Nieuwenhuis EE, VAN Der Woude CJ. Meta-analysis: Inosine triphosphate pyrophosphatase polymorphisms and thiopurine toxicity in the treatment of inflammatory bowel disease. Aliment Pharmacol Ther 2007; 26: 643-52. 